\title{
La hierba de Santiago, Jacobaea vulgaris Gartn, en textos botánicos árabes andalusíes: Observaciones fitonímicas y etimológicas
}

JOAQUÍN BUSTAMANTE COSTA

Universidad de Cádiz

\section{ESTUDIO DIACRÓNICO Y SINCRÓNICO DEL LÉXICO FITONÍMICO DEL ÁRABE ANDALUSÍ}

Los textos botánicos andalusíes se están revelando como una fuente de valiosa información no sólo sobre la vegetación ibérica precolombina sino también filológica. La cita de numerosa terminología y sinonimia en una época tan temprana como los siglos XI, XII y XIII arroja luz sobre aspectos del léxico romance de los que hasta ahora no había muchos datos. Buen número de estos datos nuevos aparecen en la enciclopedia botánica de Abū l-Hayr al-'Išbīlī titulada 'Umdat aț-țabīb fì ma'rifat an-nabāt li-kulli labīb («El apoyo del médico en el conocimiento de las plantas para todo experto») cuya laboriosa edición y traducción ha finalizado no hace muchos años ${ }^{1}$.

Como ejemplo del fecundo resultado que pueden dar los estudios filológicos de las obras botánicas andalusíes, se expone aquí el caso del fitónimo ț(a)rāšnah.

Hasta la publicación de la 'Umdah la única referencia a esta planta se encontraba en el Kitāb al-Ğāmi li-mufradāt al-'adwiyah wa-l-'ag didyah del conocido botánico malagueño emigrado a Oriente Ibn al-Bayțār, del siglo XIII, que la citaba tomándola del botánico anterior Aḥmad Ibn Muḥammad al-Ġāfiqīi, cordobés del siglo XII. La obra de al-Ġāfiq̄i la editaron Meyerhof y Sobhy entre 1932 y 1940, pero lamentablemente no la completaron y la palabra ț(a)răšnah se quedó sin edición ni estudio. Por tanto, hasta la publicación de la 'Umdah el único texto sobre este fitónimo andalusí era el de Ibn alBayțār, cuyas descripción y sinonimia se resistían a la identificación de la especie.

En la 'Umdah, que es un siglo y medio anterior a la obra de Ibn al-Bayțār, los datos son más abundantes, pues se menciona en seis ocasiones ( $\S 1788,2347,2355,3324$,

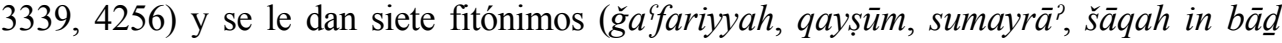

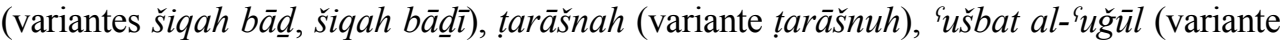

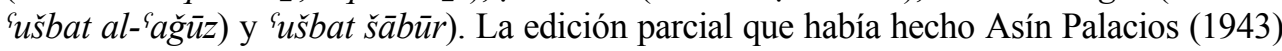
mencionaba parte de estos pasajes, especialmente aquellos donde aparecían términos de origen romance, pero ni en el interés de Asín se daba la identificación botánica (Bustamante 2005: 139) ni los datos que seleccionaba de manera muchas veces arbitraria podrían resultar suficientes para los botánicos que intentaron reconducir la identificación basándose en la fragmentaria publicación de estos textos de la ${ }^{`} U m d a h$. Font Quer (1950: 215) llegó a decir que no pocos de sus artículos había que considerar como verdaderos acerti-

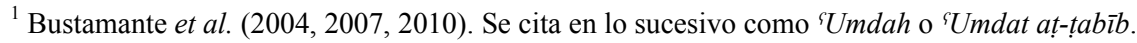


jos; por su parte Álvarez López (1947: 113) con estos datos no pudo llegar más que a una identificación errónea que más adelante se expone.

\section{COTEJO DE LOS TEXTOS RELATIVOS AL FITÓNIMO ȚARĀŠNAH}

El texto de al-Ğāfiqī que aparece citado en el Ǧămi $i^{\varsigma}$ de Ibn al-Baytậr lo cotejamos

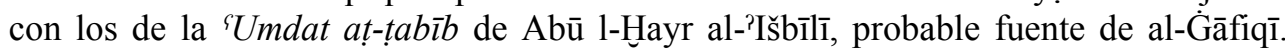
Entre todos permitirán el análisis de las descripciones botánicas para llegar a una identificación verosímil.

\subsection{Relación de pasajes de la ${ }^{\complement} \mathrm{Umdat}$ aț-țabīb donde se menciona esta planta:}

tarāšnah ${ }^{2}$ : tiene dos variedades, una de hojas como las del nabo silvestre [salğam barrī], pero más delgadas, recortadas y dentadas, rizadas, de color verde, cubiertas de algo blanco como polvo, siendo su color como el de las coles [akrunb], surgiendo de entre ellas un tallo redondo y hueco, grueso, nervado, que alcanza una altura como la talla humana, culminado por pequeñas ramas cortas en cuyos extremos hay una flor como la de la hierba pulguera [ğatğât $]$ pero mayor, crece en los lugares húmedos y las hondonadas en el tiempo de la canícula, y tiene una raíz blanca muy ramificada. Algunos pretenden que es «eléboro blanco» [harbaq abyaḍ], llamándola el vulgo ğafariyyah, gentilicio de Ğa $a^{\varsigma}$ far, que fue el primero que probó su utilidad en oftalmología, y también se llama šiqah $b \bar{a} \underline{d} \bar{l}^{3}$, es decir que uno puede segarla de pie por su talla, teniendo en consideración su penacho. La otra variedad ${ }^{4}$ tiene las hojas como las de la precedente en cuanto a la forma, pero su verde tiende a amarillo, tiene el tallo delgado, que se eleva como la altura de una persona sentada y se divide al final en muchas ramas en todas direcciones, su flor es como la de la precedente en forma, color y olor, y crece en los lugares húmedos de los prados y otros sitios. Ambas especies son plantas de verano y la primera es específico para erradicar el leucoma ocular.

'ušbat šābūr («hierba de Sapor»): es el sacapeos [ [̆a $a^{\varsigma}$ fariyyah] ${ }^{5}$.

'ušbat al-' $\boldsymbol{u} \check{\text { ğūl }}$ («hierba de terneros») ${ }^{6}$ : es el sacapeos [tarāšnuh], porque les cura el albugo de los ojos.

qayșūm ${ }^{7}:$ [...] Entre los qayāṣīm está la planta llamada šiqah bā $\underline{d}^{8}$, de hojas como las del glaucio amarillo [māmīta $\bar{a}$, pero más suaves, dentadas, recortadas y an-

\footnotetext{
${ }^{2}$ ‘Umdah, vol. II, § 2347. Corriente (2008: 213, țaraśénna).

${ }^{3}$ Corriente (2008: 210).
}

${ }^{4}$ Identificada erróneamente en el vol. II de la 'Umdah como Senecio gallicus Chaix in Vill., «árnica falsa», «cachapedo», «flor de Santiago», en realidad debe de tratarse de Jacobaea aquatica (Hill) G. Gaertn., B. Mey. y Scherb. [= Senecio aquaticus Hill], «azuzón, casanios, pericón, zacapeos», que florece en julio y agosto, como la especie Jacobaea vulgaris Gaertn. [= Senecio jacobaea L.], mientras que Senecio gallicus Chaix lo hace en primavera.

${ }^{5}$ Umdah, vol. II, § 3324.

${ }^{6}$ Gmdah, vol. II, § 3339. Es evidente que no puede ser buena la lectura عشبة العجول ('ušbat alعuğūl «hierba de los terneros») y que hay que sustituirla, como había corregido Leclerc, por عُبة العجول ('ušbat al-`ă̌uzz «hierba de las viejas») una vez establecida la identidad con la especie Jacobaea vulgaris Gaertn. [= Senecio jacobaea L.] por la sencilla razón de que los terneros, las vacas, y los demás ganados, se guardan bien de pastar jamás esta hierba porque es venenosa para los rebaños -y para los humanos-, y que la justificación del nombre que se da en el texto, que cura el albugo ocular (bayā te que se está refiriendo a seres humanos. 
chas, del verde de las hojas de la col [akrunb], con un tallo del grosor del pulgar, nervado, hueco, como de la talla humana, con ramas cortas y enhiestas, culminadas por cabezas parecidas a las de la manzanilla loca [bābūna ğ așfar $]^{9}$, con flores de color cera, y una raíz muy ramificada a partir de un pie, de color blanco; algunos pretenden que esos raigones son el eléboro blanco [harbaq abyad], lo que no es cierto. Quien quiere segar esta planta puede hacerlo de pie y erecto; por eso se llama en romance $\breve{s} \bar{a} q a h$ in $b \bar{a} \underline{d}$, que quiere decir «siega en pie»; es el tarāšnuh, en letra $t \bar{a}^{2}$, y esta planta se relaciona con la llamada $\stackrel{g}{a} a^{\varsigma} f a r i y y a h^{10}$, y también se llama «morenita» [sumayrā'], en letra sìn.

\subsection{Pasaje del Ğāmi` de Ibn al-Baytậr ${ }^{11}$ :}

Tarāăana. Hierba de Santiago: Al-Gāfiq̄i. Hay dos variedades: una que tiene las hojas parecidas a las del nabo silvestre, pero más delgadas, recortadas y rizadas, de un color verde igual al de las hojas de la col, y sobre las que hay algo blanco, parecido al polvo. El tallo adquiere la altura de un hombre, y en la parte superior tiene pequeñas ramas, en cuyos extremos nacen flores amarillas como las de la altabaca o las de la achicoria. La raíz es blanca y muy ramificada. Si se bebe el zumo de esta planta, cura la inflamación, la hidropesía y las afecciones del hígado y el bazo; su jugo también se utiliza en forma de colirio contra las cataratas. La otra variedad se parece a la primera pero su color verde tiende al amarillo, tiene el tallo más corto y más delgado, y está más ramificada. Las dos variedades crecen entre la maleza y en lugares húmedos. Es una planta estival. Esta segunda variedad también erradica las cataratas. A esta planta también se le da el nombre de $\hat{y} a^{\varsigma} f a r i y a y^{\complement} u s ̌ b a t a l-{ }^{\varsigma} a \hat{y} \bar{u} z$ porque cura las cataratas.

2.3. Tabla comparativa de los datos sobre la ț(a)rāšnah en Ibn al-Baytār (= al$\dot{G} \bar{a}$ fiqū) y Abū l-Hayr en sus dos descripciones:

\begin{tabular}{|c|c|c|c|}
\hline $\mathrm{n}^{\mathrm{o}}$ & $\begin{array}{l}\text { Ibn al-Bayțār, s. XIII (< } \\
\text { al-Ġāfiqīi, s. XII) }\end{array}$ & Abū l-Hayr, s. XI, § 2347 & $\begin{array}{l}\text { Abū l-Hayr, s. XI, } \S 4256 \\
(y \S \S 1788,3324,3339)\end{array}$ \\
\hline \multicolumn{4}{|c|}{ DESCRIPCIÓN } \\
\hline 1 & $\begin{array}{l}\text {-hojas parecidas a las del } \\
\text { nabo silvestre [salğam barrī, } \\
\text { Brassica rapa L.], pero más } \\
\text { delgadas, recortadas y riza- } \\
\text { das, de un color verde igual } \\
\text { al de las hojas de la col } \\
\text { [kurunb, Brassica oleracea }\end{array}$ & $\begin{array}{l}\text {-hojas como las del nabo } \\
\text { silvestre [salğam barrī], } \\
\text { pero más delgadas, recorta- } \\
\text { das y dentadas, rizadas, de } \\
\text { color verde, cubiertas de } \\
\text { algo blanco como polvo, } \\
\text { siendo su color como el de }\end{array}$ & $\begin{array}{l}\text {-hojas como las del glaucio } \\
\text { amarillo [māmit } \bar{t} \bar{a} \text {, Glaucium } \\
\text { flavum Crantz], pero más } \\
\text { suaves, dentadas, recortadas } \\
\text { y anchas, del verde de las } \\
\text { hojas de la col [akrunb, } \\
\text { Brassica oleracea L.] }\end{array}$ \\
\hline
\end{tabular}

${ }^{7}$ Umdah, vol. II, § 4256. Fitónimo genérico para Artemisia sp., «ajeas», «artemisas», «abrótanos», «ajenjos» y otras compuestas aromáticas similares.

${ }^{8}$ Corriente (2008: 210).

${ }^{9}$ Anthemis tinctoria $\mathrm{L}$., «manzanilla loca».

${ }^{10}$ Según $\S 2347$ no es que esté relacionada, sino que es la misma especie, por lo que cabe una traducción alternativa de la frase ويتعلق هذا النبات بنبات يدعى بالجعفرية como «y a esta planta se refiere la llamada ğa fariyyah»».

${ }^{11}$ Según la traducción de Cabo González (2009: 55), que ya incorporó la identificación con la hierba de Santiago aparecida en Abu 1-Hayr al-'Išbīlī 2007. Respetamos en la cita la transcripción que hace la traductora de $\hat{y}$ en vez de $\breve{g}$ para representar (AFI/IPA [dz]) y de $g$ en vez de $\dot{g}$ para $\dot{\varepsilon}$ (AFI/IPA [ь]). En su traducción francesa Leclerc (1881: 406-407) se abstuvo de intentar siquiera una identificación. 


\begin{tabular}{|c|c|c|c|}
\hline & $\begin{array}{l}\text { L.], y sobre las que hay algo } \\
\text { blanco, parecido al polvo }\end{array}$ & $\begin{array}{l}\text { las coles [akrunb, Brassica } \\
\text { oleracea L.] }\end{array}$ & \\
\hline 2 & $\begin{array}{l}\text {-el tallo adquiere la altura } \\
\text { de un hombre, y en la parte } \\
\text { superior tiene pequeñas } \\
\text { ramas, en cuyos extremos } \\
\text { nacen flores amarillas como } \\
\text { las de la altabaca [tabbāq, } \\
\text { Dittrichia viscosa (L.) W. } \\
\text { Greuter = Inula viscosa (L.) } \\
\text { Ait.] o las de la achicoria } \\
\text { [hindabāa, aquí Hyoseris, } \\
\text { Picris, Sonchus o Tara- } \\
\text { xacum, no Cichorium] }\end{array}$ & 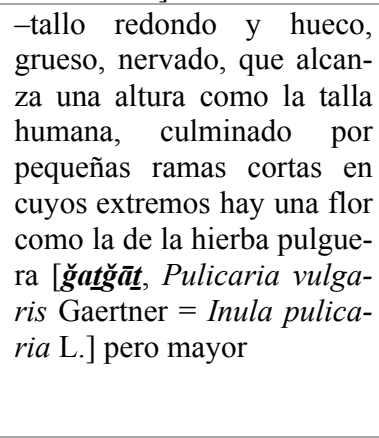 & $\begin{array}{l}\text {-tallo del grosor del pulgar, } \\
\text { nervado, hueco, como de la } \\
\text { talla humana, con ramas } \\
\text { cortas y enhiestas, culmina- } \\
\text { das por cabezas parecidas a } \\
\text { las de la manzanilla loca } \\
\text { [bābünăg asfar, Anthemis } \\
\text { tinctoria L.], con flores de } \\
\text { color cera }\end{array}$ \\
\hline 3 & $\begin{array}{l}\text {-la raíz es blanca y muy } \\
\text { ramificada }\end{array}$ & $\begin{array}{l}\text {-tiene una raíz blanca muy } \\
\text { ramificada. Algunos pretenden } \\
\text { que es «eléboro blanco» } \\
\text { [harbaq abyad, Veratrum } \\
\text { album L.] }\end{array}$ & $\begin{array}{l}\text {-raíz muy ramificada a partir } \\
\text { de un pie, de color blanco; } \\
\text { algunos pretenden que esos } \\
\text { raigones son el eléboro blan- } \\
\text { co [harbaq abyad], lo que } \\
\text { no es cierto }\end{array}$ \\
\hline 4 & $\begin{array}{l}\text {-crece entre la maleza y en } \\
\text { lugares húmedos. Es una } \\
\text { planta estival. }\end{array}$ & $\begin{array}{l}\text {-crece en los lugares húme- } \\
\text { dos y las hondonadas en el } \\
\text { tiempo de la canícula }\end{array}$ & \\
\hline \multirow[t]{2}{*}{5} & $\begin{array}{l}\text {-si se bebe el zumo de esta } \\
\text { planta, cura la inflamación, la } \\
\text { hidropesía y las afecciones } \\
\text { del hígado y el bazo; su jugo } \\
\text { también se utiliza en forma } \\
\text { de colirio contra las cataratas. }\end{array}$ & $\begin{array}{l}\text {-utilidad en oftalmología } \\
\text {-es específico para erradicar } \\
\text { el leucoma ocular }\end{array}$ & \\
\hline & \multicolumn{3}{|c|}{ SINONIMIA } \\
\hline 6 & tarāšana & $\begin{array}{l}\text { tarǟ̌snah [también mencio- } \\
\text { nada en } \S 2355]\end{array}$ & tarāšnuh \\
\hline 7 & ğa fariyya & $\begin{array}{l}\text { - llamándola el vulgo } \\
\text { ğa f́ariyyah, gentilicio de } \\
\text { Ğa`far, que fue el primero } \\
\text { que probó su utilidad en } \\
\text { oftalmología }\end{array}$ & ğa fariyyah \\
\hline 8 & 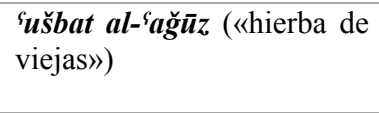 & & 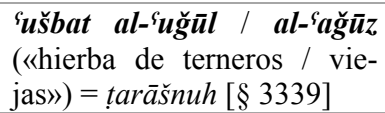 \\
\hline 9 & & & $\begin{array}{l}\text { 'ušbat šābū r («hierba de } \\
\text { Sapor») }=\check{g} a^{\varsigma} \text { fariyyah } \\
3324]\end{array}$ \\
\hline 10 & & & sumayr $\bar{a}^{2}[$ también $\S 1788]$ \\
\hline 11 & & $\begin{array}{l}\text {-también se llama šiqah } \\
\boldsymbol{b} \overline{\boldsymbol{a}} \boldsymbol{d} \overline{\bar{z}} \text {, es decir que uno puede } \\
\text { segarla de pie por su talla, } \\
\text { teniendo en consideración } \\
\text { (que lo que interesa es) su } \\
\text { penacho }\end{array}$ & 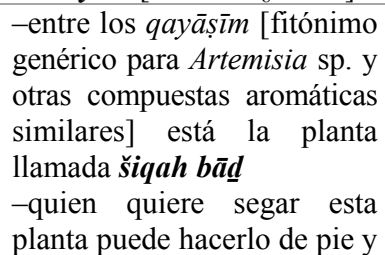 \\
\hline
\end{tabular}




\begin{tabular}{|c|c|c|c|}
\hline & & & $\begin{array}{l}\text { erecto; por eso se llama en } \\
\text { romance } \check{s} \bar{a} q a h \text { in } b \bar{a} \underline{d} \text {, que } \\
\text { quiere decir «siega en pie» }\end{array}$ \\
\hline & \multicolumn{3}{|c|}{ OTRA VARIEDAD } \\
\hline 12 & $\begin{array}{l}\text {-otra variedad se parece a la } \\
\text { primera pero su color verde } \\
\text { tiende al amarillo, tiene el } \\
\text { tallo más corto y más delga- } \\
\text { do, y está más ramificada. } \\
\text { Las dos variedades crecen } \\
\text { entre la maleza y en lugares } \\
\text { húmedos. Es una planta } \\
\text { estival. Esta segunda varie- } \\
\text { dad también erradica las } \\
\text { cataratas. A esta planta tam- } \\
\text { bién se le da el nombre de } \\
\text { ğa'fariya y 'ušbat al-'ağūz } \\
\text { porque cura las cataratas. }\end{array}$ & $\begin{array}{l}\text { - otra variedad tiene las hojas } \\
\text { como las de la precedente en } \\
\text { cuanto a la forma, pero su } \\
\text { verde tiende a amarillo, tiene } \\
\text { el tallo delgado, que se eleva } \\
\text { como la altura de una persona } \\
\text { sentada y se divide al final en } \\
\text { muchas ramas en todas direc- } \\
\text { ciones, su flor es como la de } \\
\text { la precedente en forma, color } \\
\text { y olor, y crece en los lugares } \\
\text { húmedos de los prados y } \\
\text { otros sitios. Ambas especies } \\
\text { son plantas de verano }\end{array}$ & \\
\hline
\end{tabular}

\section{JUSTIFICACIÓN DE LA IDENTIFICACIÓN BOTÁNICA}

La primera vez que se intentó una identificación botánica de esta planta lo hizo Asín Palacios (1943: 269-271) con escaso criterio y de manera incoherente, pues se limitó a poner junto al nombre romance de la ${ }^{\complement} U m d a h$ la equivalencia de dos fitónimos españoles que son incompatibles entre sí: «515. ŠECA PEDE, ŠECA PED, ŠECA EN PED = variedad de abrótano cantueso»y y en el comentario posterior lo justifica porque en el texto (línea 11 de nuestra tabla) se dice que la planta es «una especie de los qayāșìm» y encuentra en Meyerhof (1940: 337) que qayșūm, plural qayāșìm, son los abrótanos (género Artemisia), pero como también encuentra en Dozy (1881: 682) que Pedro de Alcalá (1505: 89) había recogido en el árabe granadino que al cantueso lo llamaban «morenita» [sumayrā']: «cantuesso mata conocida: çumáyra», no tiene impedimento en sumar a abrótano cantueso, quizá para que el lector elija. Esta identificación y el haber seleccionado el texto arbitrariamente, eliminado los datos de descripción de hojas, raíces, hábitat, es lo que ha desorientado a los botánicos que intentaron sacar partido a sus fragmentarias descripciones, Álvarez López (1947: 113) sugirió que «a juzgar por las referencias a su elevada talla, a sus cabezuelas de compuesta y a su inclusión en el género arábigo de los abrótanos, pudiera muy bien ser Artemisia arborescens L. [...], la mayor especie del género existente en nuestra flora». Font Quer (1950) ni siquiera lo intentó.

Pero teniendo en cuenta todos los datos que suministra la peculiar descripción que aparece en el texto establecido en la edición de 2004/2007/2010 se ha concluido por identificar la planta como la asterácea Jacobaea vulgaris Gaertn. [= Senecio jacobaea L.].

El sistema usado en la ${ }^{\varsigma} U m d a h$ para describir abunda en el recurso clásico de las comparaciones con plantas muy conocidas, usado desde Dioscórides y Plinio. En este caso se comparan las distintas partes de la llamada țarāšnah con el nabo silvestre, Brassica rapa $\mathrm{L}$. subsp. campestris (L.) A. R. Clapham, con el glaucio amarillo Glaucium flavum Crantz, con la col, Brassica oleracea L., con la altabaca, Dittrichia viscosa (L.) W. Greuter (= Inula viscosa (L.) Ait.), con la «achicoria de flor amarilla», nombre de compuestas lactucoideas 
como Hyoseris, Picris, Sonchus o Taraxacum -no Cichorium, cuya flor es notoriamente azul-, y con la manzanilla loca, Anthemis tinctoria L.

La descripción de las hojas (línea 1 de nuestra tabla) coincide con las de Jacobaea vulgaris Gaertn. [= Senecio jacobaea L.], que tiene las hojas lobuladas con el lóbulo terminal mayor, en cierto modo parecidas a las hojas inferiores del nabo silvestre $\mathrm{o}, \mathrm{y}$ en este caso más, a las del glaucio. El aspecto blanquecino pulverulento es el que toman durante la floración antes de marchitarse.

La altura, comparada con la talla humana (línea 2 de nuestra tabla), recoge bien las dimensiones de Jacobaea vulgaris Gaertn. [= Senecio jacobaea L.], que oscilan entre el metro y medio y los dos metros en condiciones óptimas.

La floración (línea 2 de nuestra tabla) en pequeñas ramas cortas, describe la disposición en corimbo y la comparación de sus flores con las de otras asteráceas como la altabaca, Dittrichia viscosa (L.) W. Greuter [= Inula viscosa (L.) Ait.] o la achicoria, que aquí sería no Cichorium, de flor azul, sino otra compuesta lactucoidea de flor amarilla como Hyoseris, Picris, Sonchus o Taraxacum, en el caso de Ibn al-Bayțār, y en el de Abū l-Hayr la hierba pulguera, Pulicaria vulgaris Gaertner [= Inula pulicaria L.], en la primera descripción, o la manzanilla loca, Anthemis tinctoria L., en la segunda, sitúa claramente la planta entre las compuestas (Asteraceae) con flores liguladas amarillas.

La descripción (línea 3 de nuestra tabla) de la raíz, blanca y ramificada a partir de una pequeña cepa es la que conviene también a la de Jacobaea vulgaris Gaertn.

El hábitat (línea 4) en terrenos húmedos y la floración estival son también característicos de Jacobaea vulgaris Gaertn. [= Senecio jacobaea L.].

Por lo que respecta a la otra especie o variedad suya (línea 12), muy probablemente se trate de Jacobaea aquatica (Hill) G.Gaertn., B.Mey. y Scherb. [= Senecio aquaticus Hill], que también comparte el hábitat de pastizales húmedos, con hojas de lóbulos similares, que comienza su floración en julio como la especie Jacobaea vulgaris Gaertn., pero es de talla algo menor.

\section{CONEXIONES ETIMOLÓGICAS}

La sinonimia corrobora la identificación con Jacobaea vulgaris Gaertn. [= Senecio jacobaea L.] y proporciona nuevos datos que pueden ayudar para establecer la etimología de algunos fitónimos, generalmente términos oscuros o camuflados.

4.1. El fitónimo que encabeza artículo, tanto en Ibn al-Bayțār como en Abū l-Hayr, taräšsana, țarāšnah, que alguna vez se encuentra vocalizada tarāănuh, puede ayudar para la identificación etimológica del fitónimo galaico-portugués tasna, tasneira, tasneirinha, tasninha ${ }^{12}$ que se emplea justamente para Jacobaea vulgaris Gaertn. [ $=\mathrm{Se}$ necio jacobaea L.], y está compartido con otras especies del mismo género. Esta palabra suele darse en los diccionarios portugueses sin etimología. A la vista de la variante erva da talasma ${ }^{13}$ se puede suponer una secuencia que partiera del andalusí țarāšna >

\footnotetext{
${ }^{12} \mathrm{http}: / /$ www.dicionarioinformal.com.br/tasneira/, http://www.priberam.pt/dlpo/tasneirinha.

${ }^{13} \mathrm{http}: / /$ www.anthos.es s.v. Senecio jacobaea L. ( $\rightarrow$ Búsquedas $\rightarrow$ Género: Senecio. Especie: jacobaea $\rightarrow$ Buscar $\rightarrow$ Senecio jacobaea $\mathrm{L} . \rightarrow$ Ficha $\rightarrow$ Nombres vernáculos) registra como gallegos árnica,
} 
talasna $>$ tasna, con el frecuente cambio de vibrante por lateral y la supresión de ésta entre vocales en portugués. Pero cuál sea el origen de țarāšna sigue oscuro: Corriente (2008: 213) había aventurado un lat. trā(n)senna «red (para cazar pájaros)», cuya relación semántica con esta planta no se ve muy clara. Otra posibilidad sería ponerla en relación con el helenismo latino tardío trōxima ${ }^{14}(<\tau \rho \omega ́ \xi \xi \mu o v ~ ' c o m e s t i b l e ')$ de Alejandro de Tralles (s. VI), con variantes trōximus (el mismo Al. Trall. y Plinio Valeriano, s. IV) y trōximon (Pablo de Egina, s. VII), que habría significado 'achicoria silvestre', Cichorium intybus L., con la suposición de un desplazamiento de sentido para denominar otra especie de la misma familia, aunque no precisamente comestible.

4.2. El sinónimo ğa $\boldsymbol{a}^{\varsigma}$ fariyya («[hierba] de Ğa $\left.{ }^{\varsigma} f a r »\right)$ lo mencionan tanto Ibn al-Bayțār como Abū l-Hayr, quien da además la explicación del gentilicio como procedente de

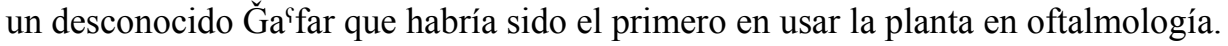

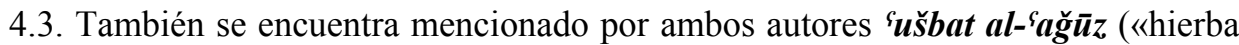

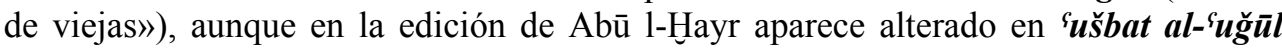
(«hierba de terneros»), cuya lectura errónea ha quedado arriba explicada en la nota 6. A finales del verano es frecuente en toda la Península ver prados esquilmados por donde han pastado rebaños de ganado vacuno que han comido todo tipo de herbaje, verde o seco, excepto los sacapeos, que suelen quedar intactos y sobresalir enhiestos más de un metro por encima de la hierba rasa.

4.4. El resto de los sinónimos que menciona Abū 1-Hayr no ha sido recogido por Ibn al-Bayțār, quizá porque no figurase ya en al-Ḡāfiqī, o quizá porque, al ser su Ğămi una obra destinada a un público oriental, considerase que se trataba de localismos excesivamente andalusíes. Uno de ellos, 'ušbat šābū 3324, se aplica tanto a Jacobaea vulgaris Gaertn. como a la «jabonera», Saponaria officinalis L., por lo que no sería de extrañar una etimología popular sobre el romance andalusí śapón «jabón» que se hubiera reinterpretado como Śābūr (Sapor), el nombre del monarca persa fundador de Gundišāpūr, habida cuenta de la similitud gráfica entre la ن [n] y la $\lrcorner$ [r] en los manuscritos árabes en letra magrebí o andalusí.

Otro, sumayr $\overline{\boldsymbol{a}}^{\text {( }}$ («morenita»), hace referencia al aspecto renegrido de la planta cuando florece el corimbo terminal mientras que todas las hojas a lo largo del tallo se encuentran ya marchitas y oscurecidas.

4.5. Pero el sinónimo más interesante desde el punto de vista filológico es el de

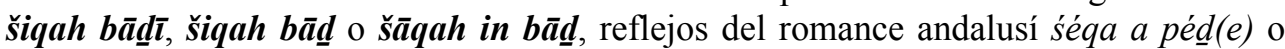
śéqa n péd (e), que, como el mismo Abū l-Hayr explica, significa «siega de pie»o «siega en pie», pues la parte aprovechable de la planta es el corimbo florido, la única parte de ella que no está marchita a finales de agosto, cuando ha desarrollado su máxima alzada, por lo que puede fácilmente recolectarse sin doblar el espinazo.

ca(s)camelo, casanio(s), casimo, cálsamo, erva-de-santiago, herba crespa / de Santiago/ do sapo / manteigueira, sacapeos, tasna y tasneira, y como portugués casanios, erva da talasma, erva-de-santiago, herba do sapo, saca-prada, sacaprada, sacapradinha, seca ossos, tasna y tasneira.

${ }^{14}$ André (1985: 265). 


\section{FORTUNA Y EVOLUCIÓN DEL FITÓNIMO ANDALUSÍ ŠIQAH BĀD(İ) [ŚÉQAPÉDE].}

El término árabe andalusí شاقه باذي fonologizado šăqah bād ción del romance andalusí [séqapéde], «siega (a) pie», sufrió con el paso a los otros romances no andalusíes del norte una adaptación consistente, en principio, en la pérdida de la -d- intervocálica, al modo castellano, que hubo de producir una forma * śeqapé(d)e antecesora de otras posteriores donde la etimología popular había de provocar un acercamiento al gallego-portugués xacobeo que introdujo el contenido semántico de «Santiago», y, por otra parte, más al sur, en ámbito extremeño, castellano manchego y andaluz occidental, también debido a etimología popular se produjo la forma sacapeos y sus variantes.

Los fitónimos vernáculos recogidos en las listas del Real Jardín Botánico de Madrid ${ }^{15}$ presentan una gran variedad de formas derivadas del andalusí séqapéde convertido en sacapeos. De entrada, esta etimología popular ha provocado que proliferen las explicaciones que le atribuyen unas virtudes inexistentes para combatir los flatos y meteorismos. Variantes del mismo nombre se han extendido a otras especies del mismo género:

Senecio aquaticus Hill: zacapeos

Senecio erucifolius L.: cachapetes, sacapeos, sacaprada

Senecio gallicus Chaix: cachapedo, cazapeto

Senecio jacobaea L.: cachapego, cachapeo, cachapero, cachapete, cachipedo, cachipeo, cachipiezo, cachipliego, cazapego, cazapegos, cazapeio, cazapete, cazapeto, cazapetos, cazapote, chacapero, galapero, gazapeo, gazapeos, gazapero, pachaquero, sacapedos, sacapeos, sacaprada ${ }^{16}$, zacapeo, zacapeos

Senecio vulgaris L.: cachapeiro, cachapete, cachapetes, cachapetina, chapaceiro, sacaprada.

La aproximación a Santiago mediante la forma xacobeo produjo los vernaculares castellanos «hierba de Santiago», «hierba jacobí» y desde la baja Edad Media se introdujeron en el latín denominaciones como herba Sancti Jacobi o herba jacobaea que recogieron los botánicos pre-linneanos de los siglos XVI y XVII creándose el nombre genérico Jacobaea que más tarde Linneo utilizó como nombre específico para esta misma planta. La explicación del nombre se quiso justificar a posteriori en que comienza su floración en julio, y el 25 de este mes se celebra entre los cristianos de rito católico la fiesta de Santiago. Otra justificación muy extendida es que se trata de una hierba que abunda en el Camino de Santiago y que por ello habría sido utilizada por los peregrinos para diversos usos medicinales. Incluso hay quien ha llegado a proponer un origen fabuloso en la isla de Santiago, en Cabo Verde ${ }^{17}$.

15 www.anthos.es s.v. Senecio jacobaea L. También sacapeos, cachapedo, hierba jacobí, y flor(es) / hierba / varita de Santiago figuran en Ceballos (1986: 653).

${ }^{16}$ Debe de ser antiguo leonés, pues Esgueva y Llamas (2005: 275) lo registran como usado en Zamora (Sanabria y la Carballeda), y en portugués se da saca-prada y sacapradinha.

${ }^{17} \mathrm{http}: / /$ www.calflora.net/botanicalnames/pageJ.html: «jacobae'a: an epithet applied to plants and deriving from two different possible sources: (1) from St. James (Jacob or Jacobus), one of the 12 Apostles; or (2) referring to the island of St. Iago in the Cape Verde Islands (ref. Senecio jacobaea)». También: Gledhill (2008). La confusión viene de que otras plantas, endemismos caboverdianos, han adoptado el 
El caso es que esta planta, con el nombre del romance andalusí séqapéde transformado en el latín botánico jacobaea, se ha acabado llamando ahora en casi todas las lenguas de Europa y su entorno con el fitónimo referido a Santiago el apóstol (herbe de Saint Jacques, erba di San Giacomo, Saint James-wort, Jakobs-Kreuzkraut, Sint-

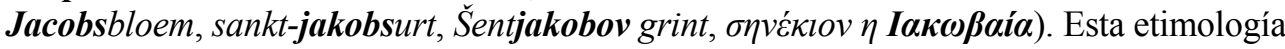
popular ha irradiado incluso hacia las lenguas de culturas no cristianas, donde para musulmanes y judíos se ha convertido en Jacob el profeta, como en turco Yakup otu, hebreo

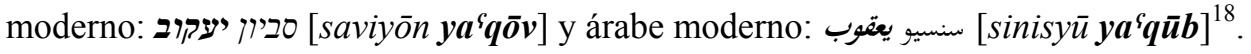

Y ya naturalizado el término jacobaea en latín botánico como nombre específico se ha desgajado de Senecio un nuevo género para el que se ha tomado Jacobaea como nombre genérico y que cuenta con un centenar de especies ${ }^{19}$, entre las cuales se encuentra la que lo originó, la que se segaba de pie que Linneo llamó Senecio jacobaea L. y ahora se prefiere llamar Jacobaea vulgaris Gaertn., la hierba de Santiago.

\section{REFERENCIAS BIBLIOGRÁFICAS}

AlCALÁ, Fray Pedro de (1505): Vocabulista arávigo en letra castellana, en Arte para ligeramente saber la lengua arábiga, Granada, Juan Varela.

ÁlVAREZ LÓPEZ, Enrique (1947): «Comentarios históricos y botánicos con motivo de un "Glosario" hispano-musulmán de los siglos XI al XII», Anales del Jardín Botánico de Madrid, 7, 11, pp. 5-175.

ANDRE, Jacques (1985): Les noms des plantes dans la Rome antique, Paris, Les Belles Lettres.

Asín PALACIOS, Miguel (1943): Glosario de voces romances registradas por un botánico anónimo hispano-musulmán (siglos XI-XII), Madrid-Granada, CSIC-Escuelas de Estudios Árabes de Madrid y Granada [edición facsímile, introducción de Vicente Martínez Tejero, Zaragoza, Institución Fernando el Católico-Universidad de Zaragoza, 1994].

Bustamante COSTA, Joaquín (2005): «Fitonimia andalusí. Etimología e identificación de la especie 'tramallah», en Jordi Aguadé, Ángeles Vicente y Leila Abu-Shams, eds., Sacrum Arabico-Semiticum. Homenaje al profesor Federico Corriente en su 65 aniversario, Zaragoza, Instituto de Estudios Islámicos y del Oriente Próximo, pp. 137-156.

Bustamante Costa, Joaquín, Federico CoRriente y Mohand Tilmatine (2004, 2007,

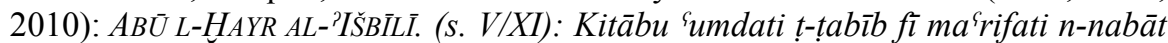
li-kulli labīb (Libro base del médico para el conocimiento de la botánica por todo experto), edición, notas y traducción castellana de... Volumen I. Fuentes arábicohispanas, n. ${ }^{\circ} 30$ [Texto árabe]. Volumen II. Fuentes arábico-hispanas, n. ${ }^{\circ} 33$. [Tra-

nombre específico jacobaea como referencia a dicha isla —por ejemplo, Campanula jacobaea C.Sm. ex Webb-, pero no es este el caso de nuestra hierba de Santiago, que no es de aquel clima.

$18 \mathrm{http}: / /$ etimologias.dechile.net/Expresiones/?Hierba-de-Santiago. La autoría de la entrada es de quien esto suscribe.

${ }^{19} \mathrm{http}: / /$ www.theplantlist.org/tpl/search?q=jacobaea . 
ducción castellana]. Volumen III (2 tomos). Fuentes arábico-hispanas, n. ${ }^{\circ} 34.1$ y 34.2) [correcciones, bibliografía e índices temáticos].

CABO GONZÁlEZ, Ana María (2009): «Una experiencia en el aula: Edición y traducción del

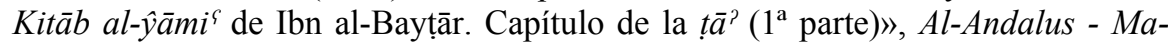
greb, 16 , pp. $45-87$.

CEBAllos JimÉnEZ, A. (1986): Diccionario ilustrado de los nombres vernáculos de las plantas en España, Madrid, ICONA.

CORRIENTE, Federico (2008): Romania Arabica. Tres cuestiones básicas: arabismos, «mozárabe» y «jarchas», Madrid, Trotta.

CORRIENTE, Federico (1997): A Dictionary of Andalusi Arabic, Leiden, Brill.

CORRIEnTE, Federico e Ignacio FERRANDo (2005): Diccionario avanzado árabe. Tomo I, árabe-español, Barcelona, Herder.

CORTÉS, Julio (1996): Diccionario de árabe culto moderno, Madrid, Gredos.

DOZY, R. (1881): Supplément aux dictionnaires arabes, Leiden, E. J. Brill.

EsGueva, Manuel y Félix Llamas (2005): El léxico de la flora silvestre en Zamora. Fitonimia y dialectología, Madrid, Velecío editores y UNED.

FonT QUER, Pío (1950): «Comentarios al "Glosario de voces romances” del Dr. Asín Palacios», Memorias de la Real Academia de Ciencias de Barcelona, 30, pp. 213-234.

GledHILL, David (2008) The Names of Plants, Cambridge, Cambridge University Press. [Cambridge Books Online. Cambridge University Press. 09 September 2010 http://dx.doi.org/10.1017/CBO9780511550898.008].

LECLERC, Lucien (1877, 1881, 1883): Traité des simples par Ibn el-Beïthar. I-III, Paris, Imprimerie Nationale.

Meyerhof, Max (1940): Šarh asmā? al-\{uqqār (L'explication des noms de drogues). Un glossaire de matière médicale composé par Maïmonide, El Cairo, Imprimerie de l'Institut Français d'Archéologie Orientale.

Meyerhof, Max y G. P. SobHy $(1932,1937,1938,1940)$ The abridged version of "The book of simple drugs" of Ahmad Ibn Muhammad al-Ghâfiqî by Gregorius Abu'lFarag (Barhebraeus) Vol. I, Fasc. I: Letter Alif. Fasc. II: Letter Bâ? and Gîm. Fasc. III: Letter Dâl. Fasc. IV: Letters Hâ and Wâw [reimpresión: Islamic Medicine, edited by Fuat Sezgin. Vols. 51-52, Frankfurt am Main, Institute for the History of Arabic-Islamic Science at the Johann Wolfgang Goethe University, 1996].

www.anthos.es

www.calflora.net/botanicalnames

www.dicionarioinformal.com.br

www.etimologias.dechile.net

www.priberam.pt

www.theplantlist.org 